\title{
Development of a Hybrid Method of CFD and Prescribed Wake Model for Helicopter BVI Noise Prediction
}

\author{
By Masahiko Sugiura, ${ }^{1)}$ Yasutada Tanabe ${ }^{1)}$ and Hideaki Sugawara ${ }^{2)}$ \\ ${ }^{1)}$ Aviation Program Group, Japan Aerospace Exploration Agency, Tokyo, Japan \\ ${ }^{2)}$ Engineering Solution Division, Ryoyu Systems Co., Ltd., Aichi, Japan
}

(Received February 4th, 2013)

\begin{abstract}
Blade vortex interaction (BVI) noise is a main source of helicopter noise especially during descending flight. Precise prediction of flow field around helicopters is required to establish the design technology of BVI noise reduction devices. Full CFD analysis including the rotor wake domain can predict flow field precisely, but it is computationally expensive. On the other hand, the prescribed wake model, which empirically predicts rotor trailing vortices, reduces computational cost greatly, but has less accuracy especially around the blade. Therefore, a hybrid method of CFD for the domain around the blade and the prescribed wake model is considered as a practical computational method in terms of the trade-off of computational accuracy and time. The base CFD code herein assumed is a structured grid Euler solver, $\langle\mathrm{rFlow} 3 \mathrm{D}\rangle$, which has been intensively developed for helicopter applications at the Japan Aerospace Exploration Agency. In this study, computational accuracy of the hybrid method is improved by applying circulation distribution, induced velocity distribution, and blade tip vortex position obtained in CFD domain to the prescribed wake model. The normal force coefficient on blade and noise contour computed by the hybrid method show good agreement with the experiment.
\end{abstract}

Key Words: Helicopter, BVI, Prescribed Wake Model, CFD

\section{Introduction}

Helicopter noise, especially blade vortex interaction (BVI) noise, is one of the environmental problems for residents around a heliport. Recently, blade airfoil and tip geometry have been developed for BVI noise reduction. On the other hand, rotor active control has also been expected to reduce BVI noise since miss distance, which is the vertical distance between the blade and the blade tip vortex, can be controlled in some flight conditions. It is very important for understanding the occurrence mechanism of BVI noise to accurately predict the behavior of the blade tip vortex, since BVI is a phenomenon that the blade tip vortex generated by a preceding blade interferes with the following one.

Several BVI noise prediction methods from high-precision CFD to simplified methods have been enthusiastically developed worldwide. ${ }^{1-7)}$ Full CFD is suited for detailed analysis, but it requires long computational time. Thus, several hybrid methods, which compute the region around a blade precisely and the wake region roughly, have been proposed for practical computational cost and accuracy. For example, the region around a blade is computed using CFD (Navier-Stokes equations ${ }^{1-4)}$ or Euler equations ${ }^{5-7)}$ ), and the wake region is computed using the Lagrangian method, ${ }^{2)}$ vortex particle method, ${ }^{3,5)}$ panel method, ${ }^{4)}$ or prescribed wake model. ${ }^{6,7)}$

In this study, Euler equations are employed for the region around a blade since it was revealed that Euler equations have sufficient computational accuracy ${ }^{8)}$ and the computa-

(C) 2013 The Japan Society for Aeronautical and Space Sciences tional cost is lower than that of Navier-Stokes equations. The prescribed wake model is selected for the wake region since it dramatically reduces computational cost by empirically defining the blade tip vortex geometry. The objective of this study is to increase computational accuracy by applying CFD information to the prescribed wake model as much as possible. In particular, circulation distribution, induced velocity distribution and the blade tip vortex roll-up position in CFD are applied to the prescribed wake model.

\section{Numerical Methods}

\subsection{Prescribed wake model}

There are several blade tip vortex models. In this study, the Vatistas $n=2$ model $^{9)}$ is employed since it agrees rather well with experiments. Figure 1 shows the Vatistas model with the Lamb-Oseen and Rankine models. Blade tip vortex models which include dissipation effect ${ }^{10)}$ accurately evaluate vortex even if the time until the blade interacts with the vortex is long. The time evolution of the vortex core radius is given as

$$
r_{c}(t)=\sqrt{r_{c 0}^{2}+4 \alpha \delta v t}
$$

where $t$ is time, $r_{c 0}$ is an initial value of vortex core radius, which is set as $20 \%$ of blade cord length, $\alpha=1.25643$, which is called the Oseen parameter, $v$ is the dynamic viscosity coefficient, and $\delta=120$, which is called the effective diffusion constant or eddy viscosity coefficient determined by the vortex Reynolds number.

Velocity is given by the Biot-Savart law using this blade tip vortex model. 


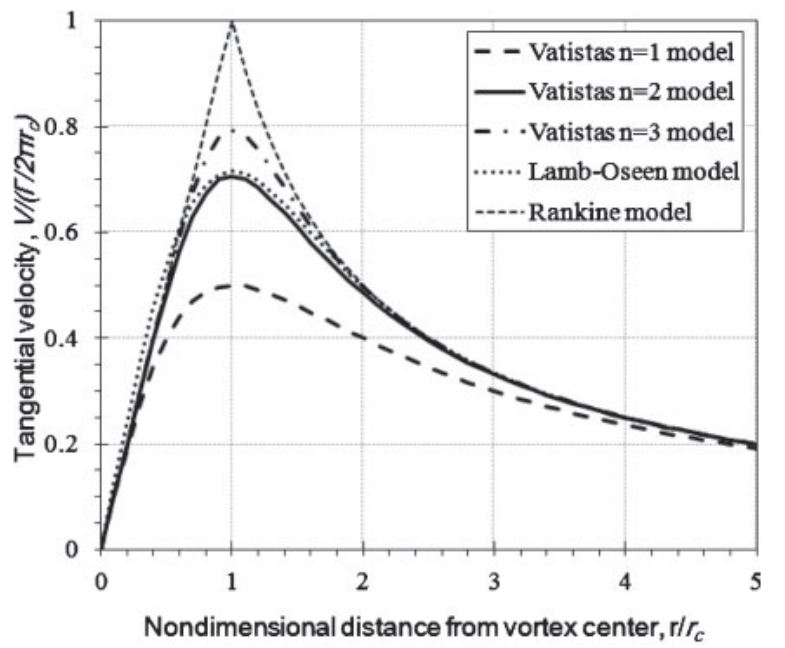

Fig. 1. Comparison of vortex wake model tangential velocity.

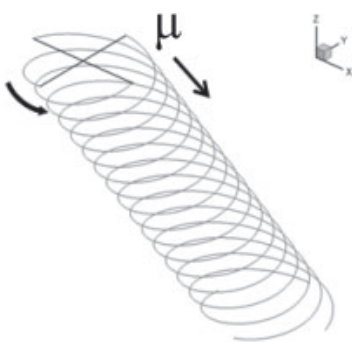

(a) Rigid model

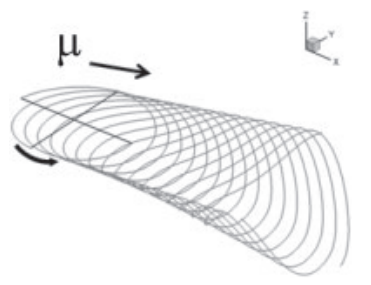

(c) Modified Beddoes's model

Fig. 2. Vortex wake trajectories of prescribed wake models.

The blade tip vortex literally generated from the blade tip flows backward spirally. In this process, the blade tip vortex moves downward gradually by rotor downwash, and the geometry of the blade tip vortex has an inclination to the rotor plane (Fig. 2).

There are some models for the prescribed wake model to describe a three-dimensional vortex geometry: the rigid model, Beddoes's model, ${ }^{11)}$ and the modified Beddoes's model $^{12)}$ (Fig. 2). The rigid model describes the blade tip vortex as a cylindrical spiral, Beddoes's model includes symmetrical roll-up of the blade tip vortex and the modified Beddoes's model reflects the asymmetrical roll-up structure of the blade tip vortex. Skew angle of the blade tip vortex is represented as $E=|\chi|$ in both Beddoes's model and the modified Beddoes's model. However, the case of $E=$ $|\chi| / 2$ is also computed since there is a report that computation with $E=|\chi| / 2$ corresponds well with experiments. ${ }^{13)}$

The modified Beddoes's model is explained below since it is the most realistic of the above prescribed wake models ${ }^{14)}$ by considering conservation of momentum and asymmetry of downwash in the horizontal direction.

The vortex trajectory projection to the $x-y$ plane is assumed as a simple epi-cycloid, and the $x-y$ coordinate is expressed as

$$
\begin{aligned}
& x_{v}=R \cos \Psi_{v}+\mu_{x} \Delta \Psi_{v} \\
& y_{v}=R \sin \Psi_{v}
\end{aligned}
$$

where $R$ is radius of rotor, $\Psi_{v}$ is blade rotation angle when the blade tip vortex is generated, $\mu_{x}=V \cos \alpha_{\mathrm{TPP}} / R \Omega$, $\Delta \Psi_{v}=\Psi_{b}-\Psi_{v}, \alpha_{\mathrm{TPP}}$ is the rotor plane's angle of attack, $\Omega$ is the blade rotation angular velocity and $\Psi_{b}$ is the blade rotation angle in computational time.

The $z$ coordinate is given as the integral of the rotor inflow's downward component and downwash $v$

$$
\begin{aligned}
z_{v} & =(1 / R \Omega) \int_{\Psi_{v}}^{\Psi_{b}}\left(-V \sin \alpha_{\mathrm{TPP}}+v\right) \mathrm{d} \Psi \\
& =\int_{\Psi_{v}}^{\Psi_{b}}\left(-\mu_{z}+v / R \Omega\right) \mathrm{d} \Psi \\
& =-\mu_{z} \Delta \Psi_{v}+\int_{\Psi_{v}}^{\Psi_{b}}(v / R \Omega) \mathrm{d} \Psi
\end{aligned}
$$

where $\mu_{z}=V \sin \alpha_{\mathrm{TPP}} / R \Omega$ and downwash $v$ is defined as (inside rotor disc)

$$
v=v_{0}\left(1+8 E / 15 \pi+E x^{\prime}-2 \mu_{x} y^{\prime}-E\left|y^{\prime 3}\right|\right)
$$

(outside rotor disc)

$$
v=2 v_{0}\left(1+8 E / 15 \pi-2 \mu_{x} y^{\prime}-E\left|y^{\prime 3}\right|\right)
$$

considering inhomogeneous downwash distribution in the longitudinal and lateral directions. The second term in the right-hand side of Eq. (3) is integrated using Eqs. (4) and (5). Variables with prime in Eqs. (4) and (5) indicate that they are normalized by rotor radius $R . v_{0}$ is given as $v_{0}=-\lambda_{I} R \Omega$, assuming uniform downwash inside the rotor disc. $\lambda_{I}$ is a solution of the following equation

$$
-C_{T} / 2 \lambda_{I}=\left[\left(\mu_{z}+\lambda_{I}\right)^{2}+\mu_{x}^{2}\right]^{1 / 2}
$$

Wake skew angle is expressed as

$$
\chi=\tan ^{-1}\left[\mu_{x} /-\left(\mu_{z}+\lambda_{I}\right)\right]
$$

\subsection{CFD}

The governing equation of CFD is a three-dimensional compressible Euler equation.

$$
\frac{\partial}{\partial t} \int_{V(t)} \boldsymbol{U} \mathrm{d} V+\int_{S(t)} \boldsymbol{F} \cdot \boldsymbol{n} \mathrm{d} S=0
$$

where $\boldsymbol{U}$ is a solution vector, $\boldsymbol{F}$ is a velocity vector for the $x$, $y$ and $z$ directions and $\boldsymbol{n}$ is a unit normal vector. Components of $\boldsymbol{U}$ and $\boldsymbol{F}$ are

$$
\boldsymbol{U}=\left(\begin{array}{c}
\rho \\
\rho \boldsymbol{v} \\
\rho e
\end{array}\right), \quad \boldsymbol{F}=\left(\begin{array}{c}
(\boldsymbol{v}-\dot{\boldsymbol{x}}) \cdot \boldsymbol{n} \rho \\
(\boldsymbol{v}-\dot{\boldsymbol{x}}) \cdot \boldsymbol{n} \rho \boldsymbol{v}+p \boldsymbol{n} \\
(\boldsymbol{v}-\dot{\boldsymbol{x}}) \cdot \boldsymbol{n} \rho \boldsymbol{e}+p \boldsymbol{v} \cdot \boldsymbol{n}
\end{array}\right)
$$

where $\boldsymbol{v}$ is a velocity vector for the $x, y$ and $z$ directions, $\dot{\boldsymbol{x}}$ is a velocity vector of the moving cell boundary, $\rho$ is air density, $p$ is pressure and $e$ is the specific total energy. 


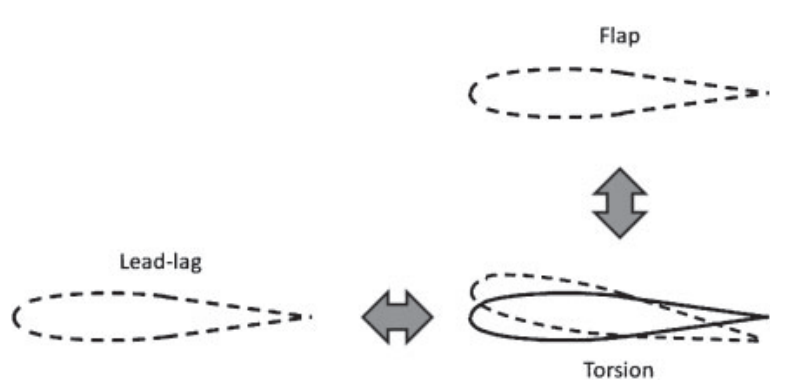

Fig. 3. Three components of blade elastic deformation.

The finite volume method and moving overlapped grid method are used in this numerical solution. The modified simple low-dissipation AUSM (mSLAU) ${ }^{15)}$ which is modified for applying an all-speed SLAU scheme to the moving overlapped grid method is used for numerical velocity, and the fourth spatial precision fourth-order compact MUSCL TVD (FCMT) scheme ${ }^{16)}$ is used for reconstruction of physical values. For time integration, the dual-time stepping method ${ }^{17)}$ is used in blade and fuselage grids to construct the unsteady implicit method. LU-SGS/DP-LUR is used for simulated time integration.

It is necessary to model blade motion precisely for accurate prediction of BVI noise since fluctuations of blade position affect the vortex generation point and configuration of the generated vortex and blade. Pitch angle control and elastic deformation moves the blade to the vertical and horizontal directions unsteadily. Blade elastic deformation has three components, vertical deformation to the blade plane (flap), horizontal movement (lead-lag), and torsion as shown in Fig. 3. Each component of blade elastic deformation is modeled.

\subsection{Hybrid method of CFD and prescribed wake model}

This hybrid method reduces computational cost by computing only around a blade in CFD, and the wake region is computed by the prescribed wake model, assuming wake region is potential flow. Density, momentum and energy in CFD are computed by reflecting the induced velocity of the blade tip vortex which is calculated by the prescribed wake model. In particular, induced velocity of the prescribed wake model is added to three outer layer grids in CFD to satisfy mass conservation law since the effect of compression is small in the area far from the blade. ${ }^{18)}$ Configuration of CFD computational grids and the prescribed wake model is shown in Fig. 4.

At the interface of CFD and the prescribed wake model, variables of flow field are calculated by the following equations. $^{18)}$

$$
\begin{aligned}
& \left(\begin{array}{l}
u \\
v \\
w
\end{array}\right)=\left(\begin{array}{c}
u_{\infty}+u_{w} \\
v_{\infty}+v_{w} \\
w_{\infty}+w_{w}
\end{array}\right) \\
& \rho=\left(\frac{a^{2}}{a_{\infty}^{2}}\right)^{\frac{1}{\gamma-1}} \\
& e=\rho\left[\frac{a^{2}}{\gamma(\gamma-1)}+\frac{u^{2}+v^{2}+w^{2}}{2}\right]
\end{aligned}
$$

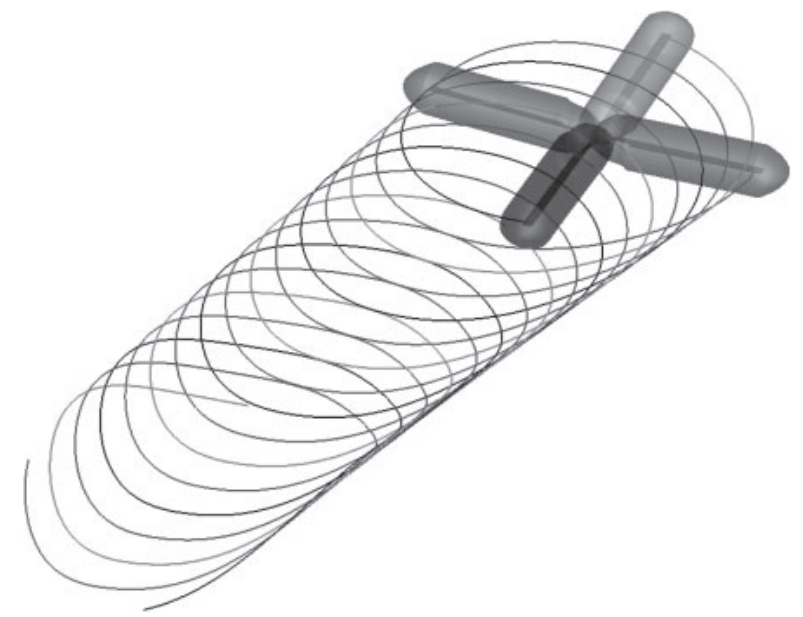

Fig. 4. Configuration of CFD computational grids and the prescribed wake model.

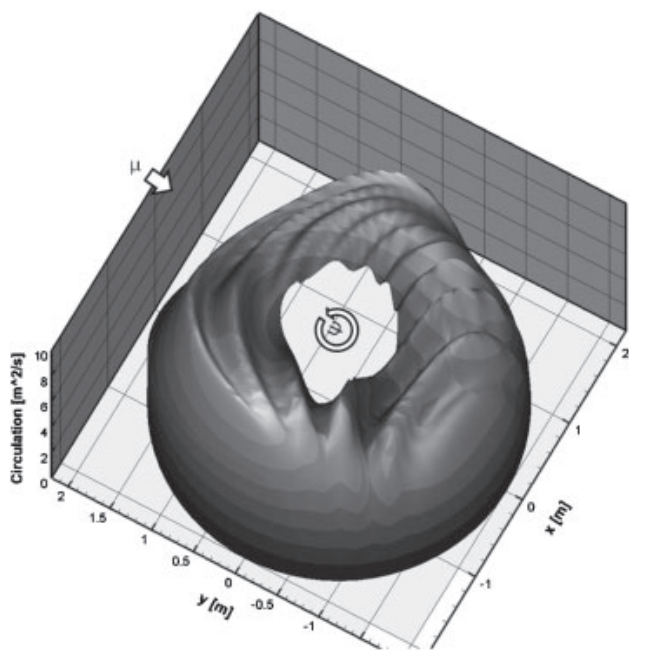

Fig. 5. Circulation distribution on blade.

where $u, v$ and $w$ are flow velocities of the $x, y$ and $z$ directions, respectively, $a$ is sound speed, $\gamma$ is the specific heat ratio, $\infty$ means the component of uniform flow, and subscript $w$ means the component of wake. Local sound speed is determined from the following energy equation.

$$
a^{2}=a_{\infty}^{2}+\frac{\gamma-1}{2}\left[V_{\infty}^{2}-\left(u^{2}+v^{2}+w^{2}\right)\right]
$$

where $V_{\infty}$ is the velocity of the main stream.

Circulation of the prescribed wake model has been set constant in the previous studies; however, circulation fluctuates greatly according to the azimuth angle (Fig. 5). In this study, circulation of the prescribed wake model is varied using circulation distribution in CFD. Here, circulation is normalized by satisfying the following equation ${ }^{13)}$ for induced velocity distribution in a rotor plane (Fig. 6) to satisfy momentum theory in forward flight.

$$
T=\int_{\mathrm{TPP}} 2 \rho A v_{i} \sqrt{\left(V_{\infty} \cos \alpha_{\mathrm{TPP}}\right)^{2}+\left(V_{\infty} \sin \alpha_{\mathrm{TPP}}+v_{i}\right)^{2}} \mathrm{~d} S
$$




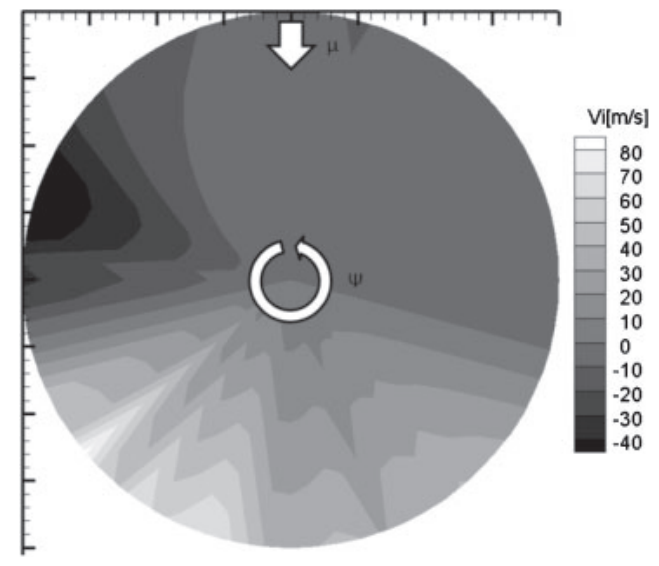

Fig. 6. Induced velocity distribution on a rotor plane.

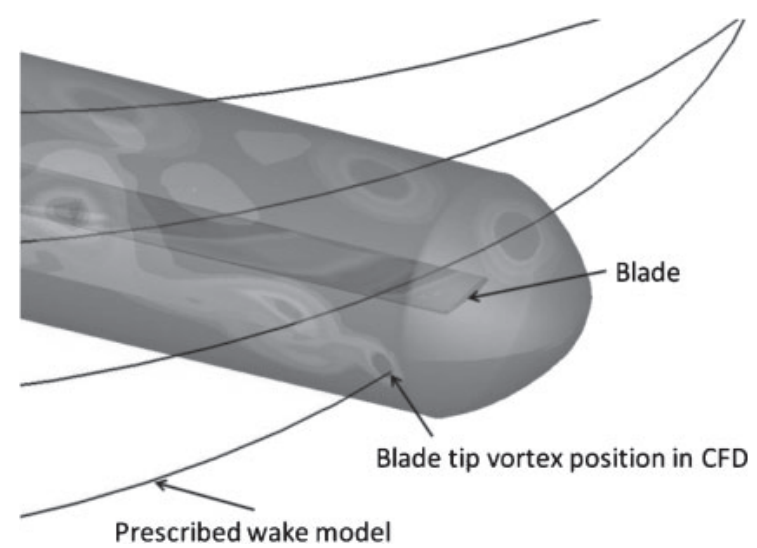

Fig. 7. Modification of the prescribed wake model using blade tip vortex positions in CFD.

where $T, A$ and $v_{i}$ represent rotor thrust, rotor disc area and induced velocity, respectively. TPP means integration is conducted on the tip pass plane. As a result, collective pitch angle agrees well with the experiment (Fig. 13(a)).

Furthermore, the initial position of the prescribed wake model is located in the blade tip vortex position in CFD as shown in Fig. 7 to capture the blade tip vortex roll-up. The vortex position in the fourth inner layer grid from the outer boundary, which is closest to the interface of CFD and prescribed wake model, is utilized to reflect the blade tip vortex roll-up growth. Blade cyclic motion is represented using this blade tip vortex position on each azimuth angle.

\subsection{Acoustic analysis}

Noise is calculated using the blade surface pressure distribution, elastic deformation, and rotor control angle of one rotor revolution obtained in CFD analysis, since steady flight condition is assumed to have periodicity synchronized with rotor revolution. Farassat's Formulation $1^{19)}$ which is a solution of sound wave equation based on Lighthill's acoustic analogy is used for acoustic analysis. This equation gives sound pressure fluctuation $p^{\prime}$ at any observation point as

$$
4 \pi p^{\prime}(\boldsymbol{x}, t)=4 \pi\left(p_{T}^{\prime}(\boldsymbol{x}, t)+p_{L}^{\prime}(\boldsymbol{x}, t)\right)
$$

Components of sound pressure are

$$
\begin{aligned}
4 \pi p_{T}^{\prime}(\boldsymbol{x}, t)= & \frac{\partial}{\partial t} \int_{f=0}\left[\frac{\rho_{0} v_{n}}{r\left(1-M_{r}\right)}\right]_{\mathrm{ret}} \mathrm{d} S \\
4 \pi p_{L}^{\prime}(\boldsymbol{x}, t)= & \frac{1}{a} \frac{\partial}{\partial t} \int_{f=0}\left[\frac{p \cos \theta}{r\left(1-M_{r}\right)}\right]_{\mathrm{ret}} \mathrm{d} S \\
& +\int_{f=0}\left[\frac{p \cos \theta}{r^{2}\left(1-M_{r}\right)}\right]_{\mathrm{ret}} \mathrm{d} S
\end{aligned}
$$

where $f=0$ means integration on the blade surface, ret is an abbreviation of "retarded time" and integration is conducted by sound source time $\tau$ which is propagated to observation point by sound speed $a$ at observation time $t . p^{\prime}$ is sound pressure to be obtained, $p_{T}^{\prime}$ is called "thickness noise," which is generated by the air pushed away by the blade motion, and $p_{L}^{\prime}$ is called "loading noise," which is generated by pressure fluctuations on the blade surface. $\rho_{0}$ is air density of uniform flow, $v_{n}$ is the vertical velocity on the surface cell, $\boldsymbol{r}=|\boldsymbol{x}-\boldsymbol{y}|$ is the distance between observation point and sound source cell, and $M_{r}=\hat{\boldsymbol{r}}_{i} \cdot \boldsymbol{v}_{i} / a$ is the sound source cell Mach number of radiation direction at sound source time. $\cos \theta$ is the cosine of the crossing angle between the vertical direction of the sound source cell and the direction from the sound source to observation point.

Sound source time (phase angle) $\tau$ satisfying the following equation, from which retarded time is obtained, must be searched on each cell of the blade surface to integrate the above equations.

$$
|\boldsymbol{x}-\boldsymbol{y}(\boldsymbol{\eta}, \tau)|=a(t-\tau)
$$

where $\boldsymbol{x}$ is the observation position, $\boldsymbol{y}$ is the sound source position, and $\boldsymbol{\eta}$ is a local coordinate on the rotor blade. $\boldsymbol{y}$ is a representative cell position divided on the blade surface, which is analytically obtained by seven steps of coordinate transformation (elastic deformation in three axis directions, feathering, flapping, lead-lag and rotation) from the blade form used in CFD. The plane vector on the surface cell is obtained by calculating the four corner coordinates of a cell.

BVISPL (sound pressure level) is defined as follows using a spectral component of sound pressure, in order to evaluate the obtained sound pressure time waveform, especially noise amplitude generated by blade and blade tip vortex interaction in intermediate frequency.

$$
\mathrm{BVISPL}=20 \cdot \log \left(\sqrt{\sum_{i=6 \times \mathrm{NBLD}}^{40 \times \mathrm{NBLD}} p_{i}} \cdot \frac{1}{p_{\text {ref }}}\right)
$$

where NBLD is the number of blades and $p_{\text {ref }}=$ $2.0 \times 10^{-5}[\mathrm{~Pa}]$.

\section{Numerical Conditions}

The precision of the hybrid method is verified by comparing the computational results with the experimental data of the higher harmonic control aeroacoustics rotor test II (HART II). ${ }^{20-22)}$ HART II is an international cooperation project of rotor active noise reduction technology and it shows that higher harmonic control of the rotor can reduce 


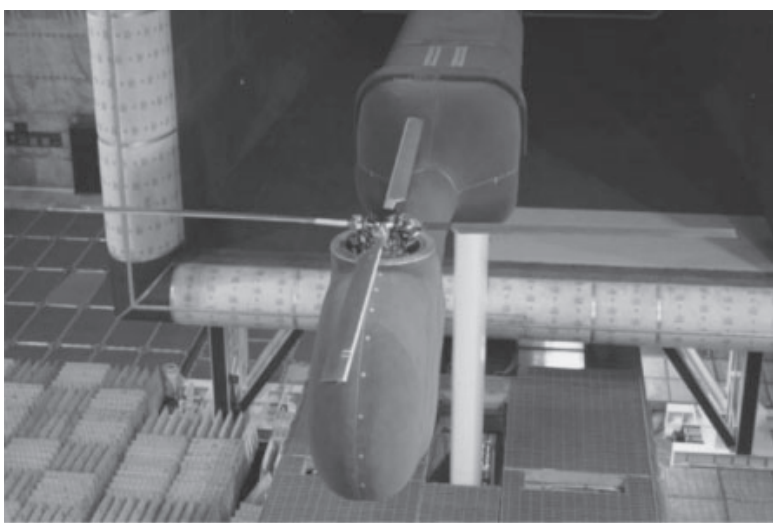

Fig. 8. HART II experiment setup. ${ }^{21)}$

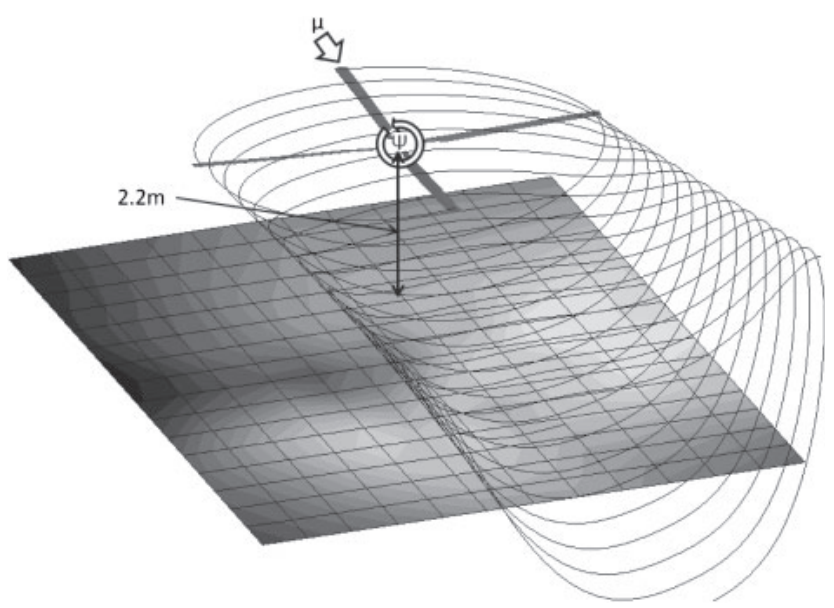

Fig. 9. Configuration of rotor and noise measurement plane of HART II experiment.

Table 1. Blade specifications.

\begin{tabular}{ll}
\hline Number of blades & 4 \\
Rotor radius & $2.0 \mathrm{~m}$ \\
Airfoil & NACA23012 \\
Chord length & $0.121 \mathrm{~m}$ \\
\hline
\end{tabular}

noise or vibration. The rotor configuration in the experiment is shown in Figs. $8^{9)}$ and 9. The noise measurement plane is located $2.2 \mathrm{~m}$ below the rotor (Fig. 9). Other experimental conditions used in this numerical analysis are explained below.

The blade specifications are shown in Table 1. Baseline conditions (Table 2) are employed in this study, and there are baseline/minimum noise/and minimum vibration conditions in the experiment. $M_{\infty}$ is the Mach number of the uniform flow, $M_{\text {tip }}$ is the Mach number of the blade tip, $\mu$ is the advancing ratio, $C_{T}$ is the rotor thrust coefficient, $C_{M X}$ is the rotor hub rolling moment coefficient, $C_{M Y}$ is the rotor hub pitching moment coefficient, and $\theta$ is the pitch angle of the blade which is expressed as the following first harmonic oscillation.

$$
\theta(\Psi)=\theta_{0}+\theta_{1 c} \cos \Psi+\theta_{1 s} \sin \Psi
$$

where $\Psi$ is the azimuth angle, $\theta_{0}$ is the collective pitch
Table 2. Baseline conditions.

\begin{tabular}{ll}
\hline$M_{\infty}$ & 0.09628 \\
$M_{\text {tip }}$ & 0.6387 \\
$\mu$ & 0.1508 \\
$C_{T}$ & $4.63 \mathrm{E}-03$ \\
$C_{M X}$ & $2.81 \mathrm{E}-05$ \\
$C_{M Y}$ & $-2.81 \mathrm{E}-05$ \\
$\theta_{0}$ & $3.800 \mathrm{deg}$ \\
$\theta_{1 c}$ & $1.916 \mathrm{deg}$ \\
$\theta_{1 s}$ & $-1.342 \mathrm{deg}$ \\
\hline
\end{tabular}
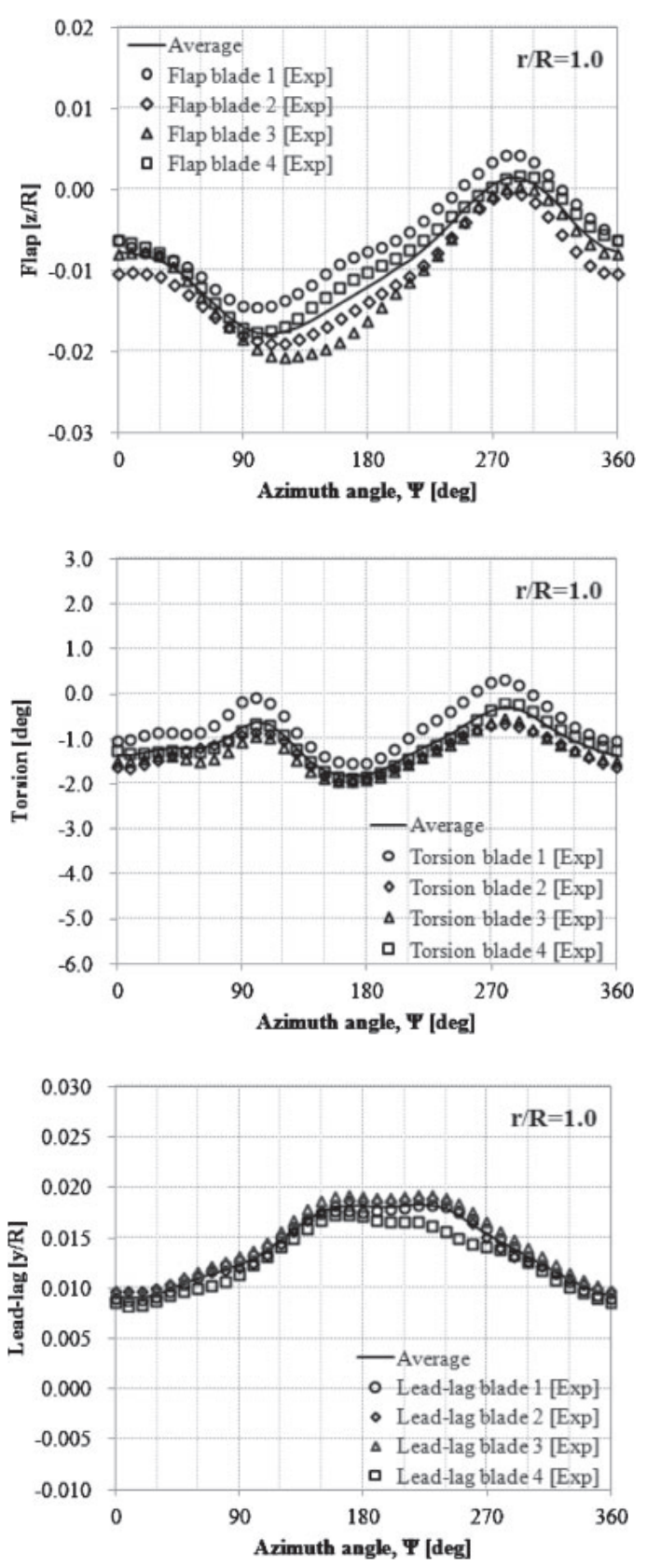

Fig. 10. Blade elastic deformation in HART II experiment.

angle, $\theta_{1 c}$ is the lateral cyclic pitch angle, and $\theta_{1 s}$ is the longitudinal cyclic pitch angle.

The blade elastic deformation is given as a function of blade spanwise distance and azimuth angle, which is an average of four measured blades' deformation (Fig. 10). 


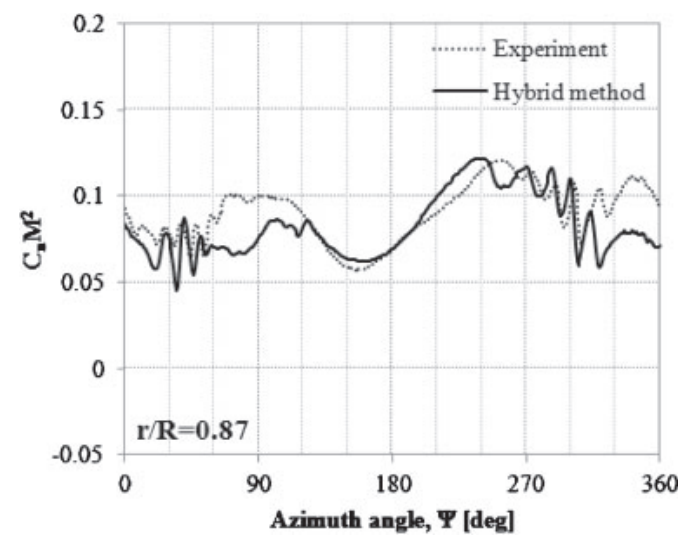

(a) Airload coefficient on a blade

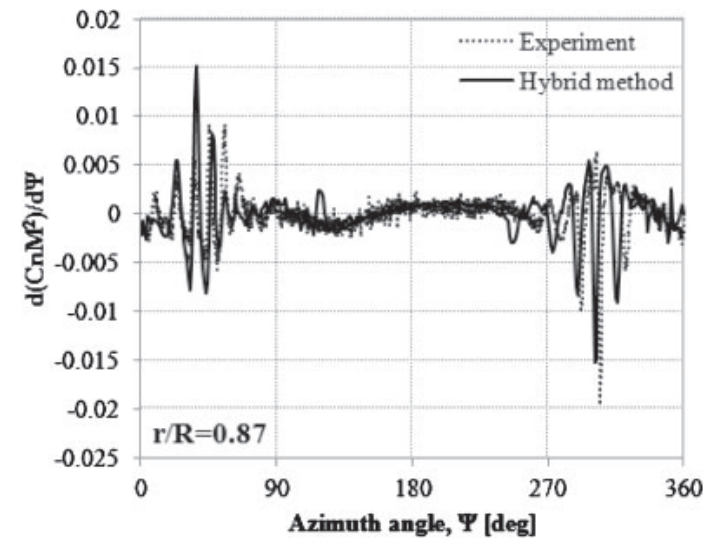

(b) Time derivative of airload coefficient on a blade

Fig. 11. Airload distribution on a blade.

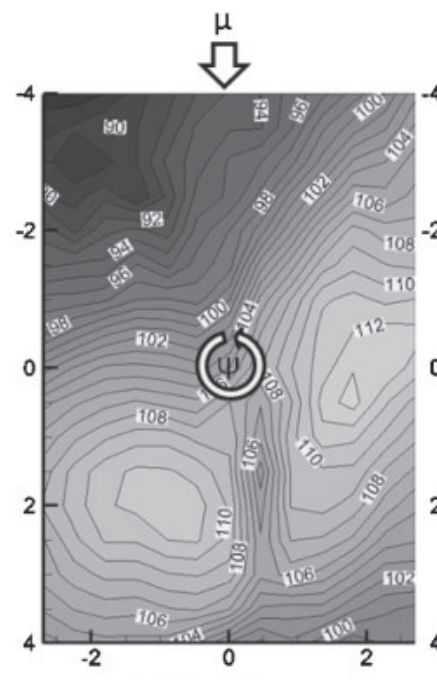

(a) Experiment

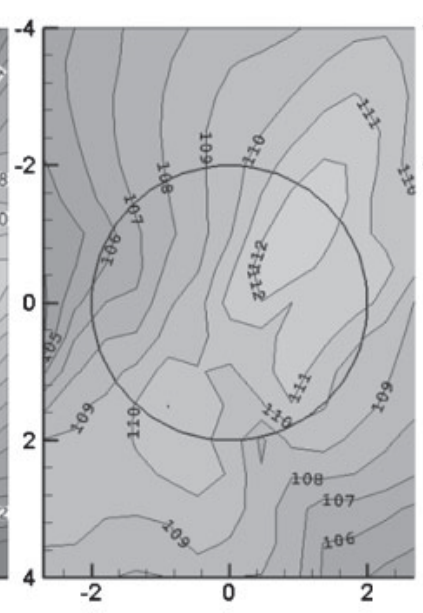

(b) rFlow3D

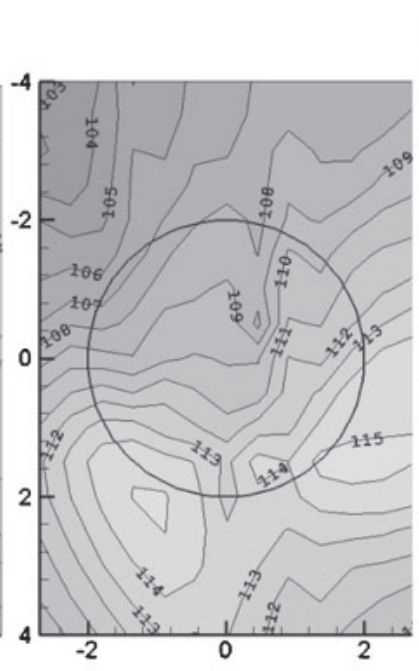

(c) Hybrid method

Fig. 12. Comparison of BVI noise distributions.

\section{Numerical Results and Discussions}

Pressure fluctuations on the blade surface computed by the hybrid method are verified. Figure 11(a) shows time variation of airload coefficient $\left(C_{n} M^{2}\right)$ at $87 \%$ span based on pressure distribution on the blade surface, and Fig. 11(b) shows the time derivative of that. From Fig. 11(a) and (b), it is found that the hybrid method predicts blade vortex interference position precisely, though it still overestimates pressure fluctuations a little on the advancing side.

BVI noise distribution computed by pressure fluctuations around the rotor is also shown in Fig. 12. HART II experimental results and computational results using full CFD (rFlow3D developed at JAXA) are also shown for comparison. It is found that noise peak positions are slightly different and sonic pressure level of the hybrid method is globally overestimated. Peak position difference is due to trim angle difference (Fig. 13(a)-(c)). Collective pitch angle indicates induced velocity, and cyclic pitch angles indicate induced velocity inclination in the horizontal and vertical directions.
Figure 14 shows the comparison of maximum BVI noise. Maximum BVI noise of the hybrid method is approximately $2 \mathrm{~dB}$ larger than that of the experiment. Conversely, that of full CFD is approximately $0.7 \mathrm{~dB}$ smaller. The reasons are considered to be that vortex positions are different between the prescribed wake model and full CFD (Fig. 15) and the vortex of the prescribed wake model interferes with the blade without decay. On the other hand, the vortex decays numerically in full CFD since the grid size is not small enough to resolve a vortex.

The hybrid method of CFD and prescribed wake model is developed and its computational time reduces to onefifteenth of that of full CFD (Table 3). Its computational accuracy is also evaluated using HART II experimental data. From Figs. 4 and 5, it is observed that the hybrid method shows good agreement with the experiment, though there are some regions of overestimating BVI noise and differences of noise peak positions. Part of the reason is assumed to be that the prescribed wake model locates the vortex near the blade and the vortex interferes with the blade 


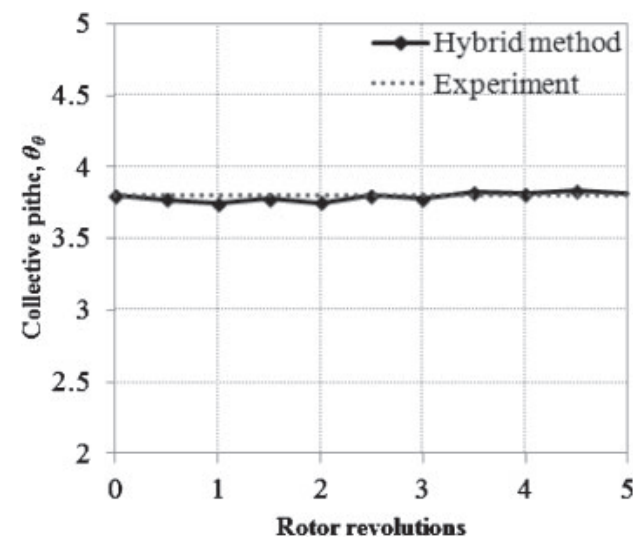

(a) Convergence of collective pitch angle

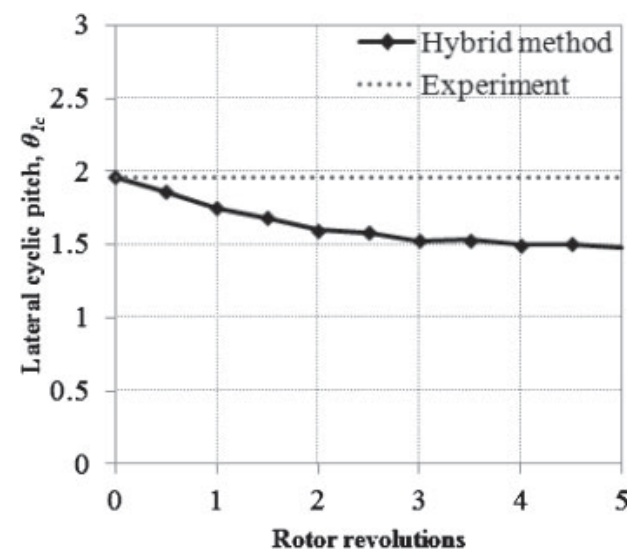

(b) Convergence of lateral cyclic pitch angle

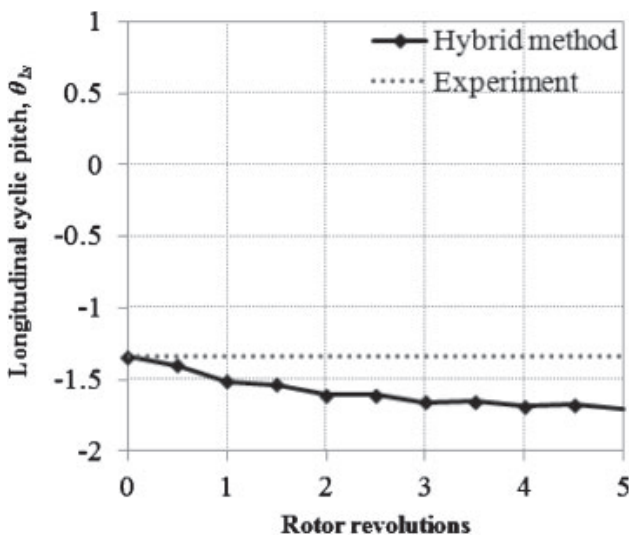

(c) Convergence of longitudinal cyclic pitch angle

Fig. 13. Convergence of trim angles.

without decay. When a vortex interferes with a blade, the vortex decays and moves in the horizontal and vertical directions.

\section{Concluding Remarks}

A hybrid method of CFD and prescribed wake model was constructed by applying CFD information to the prescribed wake model as much as possible. Computational time of the hybrid method decreased to one-fifteenth of that of full CFD. Computational accuracy of the hybrid method also

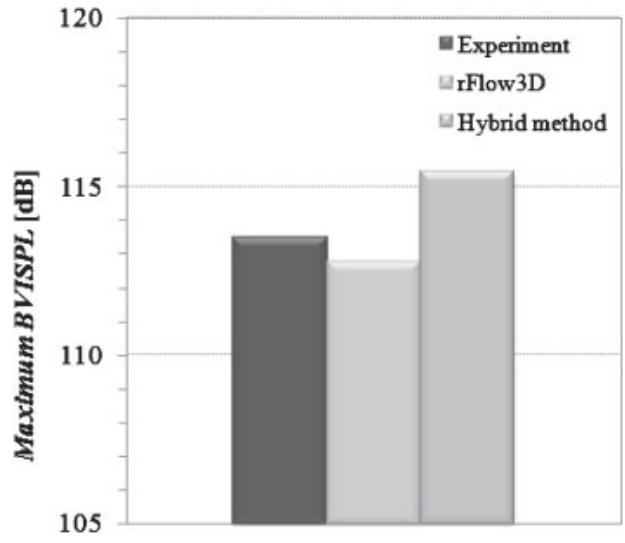

Fig. 14. Comparison of maximum BVI noise.

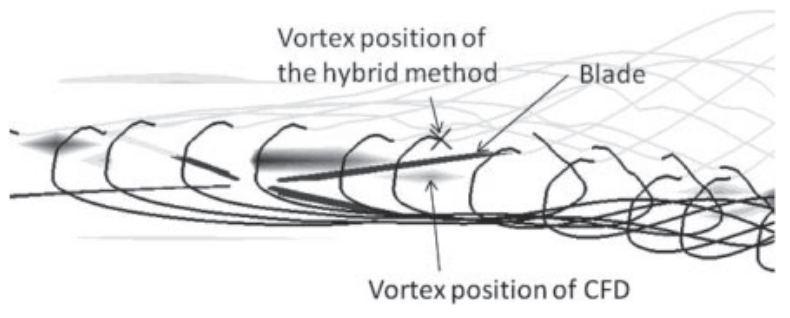

Fig. 15. Comparison of vortex positions $(\Psi=30 \mathrm{deg})$.

Table 3. Computational time using personal computer. (CPU: Corei7 (3.20 GHz), 4/8 Core, Memory: 3.0 GB)

\begin{tabular}{ll} 
rFlow3D & Approx. 30 days \\
Hybrid method & Approx. 2 days \\
\hline
\end{tabular}

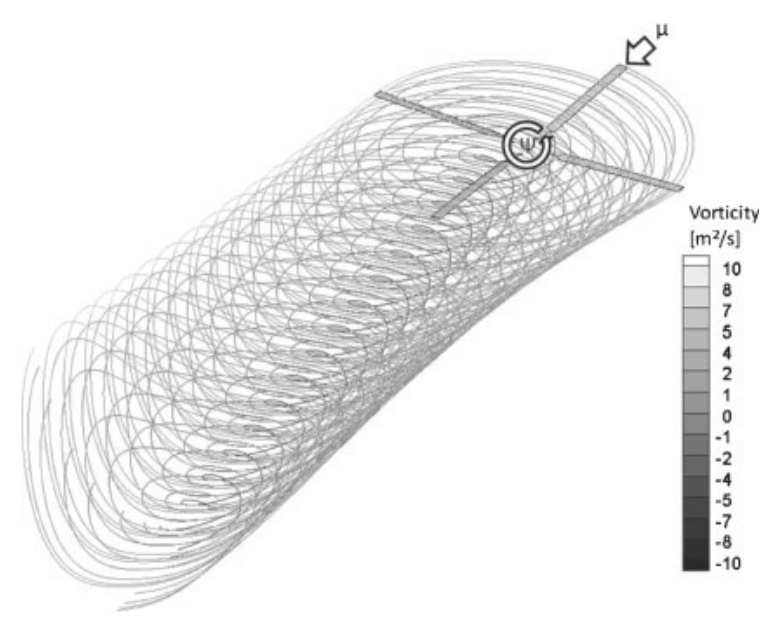

Fig. 16. Multiple trailing vortices.

showed good agreement with the experiment. However, BVI noise was overestimated on the advancing side. The reasons are considered to be that vortex positions were different between the prescribed wake model and full $\mathrm{CFD}$, and the vortex interferes with the blade without decay in the prescribed wake model. Thus, it is planned to increase computational accuracy by constructing vortex decay model in the near future. 
Trailing vortices were described as a single blade tip vortex in this study. As a result of this simplification, the effect of trailing vortices may have been overestimated. Detailed induced velocity distribution would be obtained using multiple trailing vortices. Thus, multiple trailing vortices are also planned to be employed in the near future (Fig. 16).

\section{References}

1) Boyd, Jr., D. D.: HART-II Acoustic Predictions Using a Coupled CFD/CSD Method, 65th Annual Forum of the AHS, 2009.

2) Yongjie, S., Qijun, Z. and Guohua, X.: Single-blade Based Hybrid CFD Method for Rotor Aerodynamics and Aeroacoustics Prediction, 1st Asian Australian Rotorcraft Forum and Exhibition 2012, 2012.

3) Zhao, J. and He, C.: Coupled CSD/CFD and Viscous Vortex Particle Method for Rotorcraft Comprehensive Analysis, American Helicopter Society 68th Annual Forum, 2012.

4) Park, S. H. and Lee, D.-J.: Development of a Coupled 2-D Panel/Euler Solver for Numerical Analysis of Transonic Flow, 1st Asian Australian Rotorcraft Forum and Exhibition 2012, 2012.

5) Duque, E. P. N., Stone, C. P., Hennes, C. C. and Gharakhani, A.: A Hybrid Vortex-Particle Method for Rotorcraft Applications, 30th AIAA Applied Aerodynamics Conference, 2012.

6) Inada, Y., Yang, C., Iwanaga, N. and Aoyama, T.: Efficient Prediction of BVI Noise Using Euler Solver with Wake Model, 1st International Forum on Rotorcraft Multidisciplinary Technology, 2007.

7) Lee, J., Chae, S., Oh, S. and Yee, K.: BVI Prediction Using CFDWake Coupling Method, 1st Asian Australian Rotorcraft Forum and Exhibition 2012, 2012

8) Tanabe, Y., Saito, S. and Sugawara, H.: Evaluation of Rotor Noise Reduction by Active Devices Using a CFD/CSD Coupling Analysis Tool Chain, 1st Asian Australian Rotorcraft Forum and Exhibition 2012, 2012.

9) Vatistas, G. H., Kozel, V. and Mih, W. C.: A Simpler Model for
Concentrated Vortices, Exp. Fluids, 11 (1991), pp. 73-76.

10) Bhagwat, M. J. and Leishman, J. G.: Generalized Viscous Vortex Model for Application to Free-Vortex Wake and Aeroacoustic Calculations, AHS 58th Annual Forum Proceedings, 2002.

11) Beddoes, T. S.: A Wake Model for High Resolution Airloads, 2nd International Conference on Basic Rotorcraft Research, 1985.

12) van der Wall, B. G.: The Effect of HHC on the Vortex Convection in the Wake of a Helicopter Rotor, Aerospace Sci. Technol., 4 (2000), pp. 321-336.

13) Leishman, J. G.: Principles of Helicopter Aerodynamics (second ed.), Cambridge University Press, New York, 2006, p. 612.

14) Sugiura, M., Tanabe, Y., Saito, S., Sugawara, H., Ohshio, K. and Kanazaki, M.: Hybrid Method of CFD and Prescribed Wake Model for Rotorcraft Aeroacoustics and Aerodynamics Prediction, 38th European Rotorcraft Forum, 2012.

15) Tanabe, Y. and Saito, S.: Significance of All-Speed Scheme in Application to Rotorcraft CFD Simulation, 3rd International Basic Research Conference on Rotorcraft Technology, 2009.

16) Yamamoto, S. and Daiguji, H.: High-Order-Accurate Upwind Schemes for Solving the Compressible Euler and Navier-Stokes Equations, Comput. Fluids, 22, 2/3 (1993), pp. 259-270.

17) Zhang, L. P. and Wang, Z. J.: A Block LU-SGS Implicit Dual Timestepping Algorithm for Hybrid Dynamic Meshes, Comput. Fluids, 33 (2004), pp. 891-916.

18) Yang, Z., Sankar, L. N., Smith, M. J. and Bauchau, O.: Recent Improvements to a Hybrid Method for Rotors in Forward Flight, J. Aircraft, 39, 5 (2002), pp. 804-812.

19) Farassat, F.: Derivation of Formulations 1 and 1A of Farassat, NASA/ TM-2007-214853, 2007.

20) Bailly, J., Delrieux, Y. and Beaumier, P.: Experimental Analysis and Validation of ONERA Methodology for the Prediction of BladeVortex Interaction, 30th European Rotorcraft Forum, 2004.

21) Boyd, Jr., D. D.: HART-II Acoustic Predictions Using a Coupled CFD/CSD Method, 65th Annual Forum of the AHS, 2009.

22) van der Wall, B. G. and Burley, C. L.: 2nd HHC Aeroacoustics Rotor Test (HART II) - Part II: Representative Results-, DLR 1 B 1112005/3, 2005. 